\title{
Optimal Robust Control for Bipedal Robots through Control Lyapunov Function based Quadratic Programs
}

\author{
Quan Nguyen and Koushil Sreenath
}

\begin{abstract}
This paper builds off of recent work on rapidly exponentially stabilizing control Lyapunov functions (RES-CLF) and control Lyapunov function based quadratic programs (CLFQP) for underactuated hybrid systems. The primary contribution of this paper is developing a robust control technique for underactuated hybrid systems with application to bipedal walking, that is able to track desired trajectories with significant model perturbation (mass and inertia increased by up to $200 \%$.) We evaluate our proposed control design on a model of RABBIT, a five-link planar bipedal robot.
\end{abstract}

\section{INTRODUCTION}

There is tremendous interest in employing legged and humanoid robots for dangerous missions in disaster and rescue scenarios. This is evidenced by the ongoing grand challenge in robotics, The DARPA Robotics Challenge (DRC). Such time and safety critical missions require the robot to operate swiftly and stably while dealing with high levels of uncertainty and large external disturbances. In addition to inaccurate robot models, model uncertainty can also arise from interaction tasks. For instance, practical robotic applications involving walking robots that lift and carry large unknown loads, pull unknown weights, drag heavy hoses, etc., will have to deal with significant changes to the dynamical model. The limitation of current research, as well as the demand of practical requirement, motivates our research on robust control for hybrid systems in general and bipedal robots in particular.

In recent years, the method of Hybrid Zero Dynamics (HZD), [1, 2], has been very successful in dealing with the hybrid and underactuated dynamics of legged locomotion. This method is characterized by choosing a set of output functions, which when driven to zero, creates a lowerdimensional time-invariant zero dynamics manifold. Stable periodic orbits designed on this lower-dimensional system are then also stable orbits for the full system under an appropriate controller. Until recently, experimental implementations of the HZD method relied on input-output linearization with PD control to drive the system to the zero dynamics manifold, for instance see dynamic walking [3] and running [4] on MABEL. However, recent work on control Lyapunov function (CLF)based controllers has enabled effective implementations of

This work is supported by CMU Mechanical Engineering startup funds. Q. Nguyen is with the Department of Mechanical Engineering, Carnegie Mellon University, Pittsburgh, PA 15213, email: quannguyen@ cmu.edu.

K. Sreenath is with the Depts. of Mechanical Engineering, Robotics Institute, and Electrical and Computer Engineering, Carnegie Mellon University, Pittsburgh, PA 15213, email: koushils@cmu.edu.

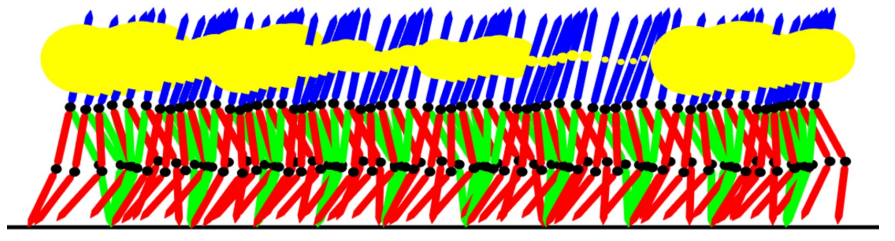

Fig. 1: A practical application of walking robots is to carry and transport large loads over diverse terrain. However, for dynamic walking, the mass of the load needs to be known for obtaining a perfect model for control. The proposed robust controller is able to demonstrate dynamic walking with a large unknown load whose mass varies at each step. Ten steps of walking are illustrated in the stick figure with the load (yellow circle) varying randomly between $0-60 \mathrm{~kg}$, corresponding to carrying an additional $0-188 \%$ of the mass of the robot.

stable walking, both in simulations and experiments [5]. This flexible control design, based on Lyapunov theory, has also enabled computing the control based on online quadratic programs (QPs), facilitating incorporating additional constraints into the control computation. For instance, control Lyapunov function based quadratic programs (CLF-QPs) with constraints on torque saturation were demonstrated experimentally in [6], and CLF-QPs were used to design a unified controller for performing locomotion and manipulation tasks in [7]. Sufficient conditions for Lipschitz continuity of the control produced by solving the CLF-QP problem are reported in [8].

However, a primary shortcoming of all these controllers is that they assume perfect knowledge of the dynamic model. The goal of this paper is to relax the requirement of perfect knowledge of the model by allowing for bounded uncertainty, and designing an optimal robust controller through a CLF-QP so as to still retain stability.

Robust control is an extensively studied topic. For robust control of linear systems, we have well established methods, such as $H_{\infty}$-based robust control and linear quadratic Guassian (LQG) based robust control [9, 10]. Additionally, a robust optimal control scheme developed by Lin [11, 12, 13] presented uncertainties in the cost functional of the optimization based on the LQR problem.

For robust control for nonlinear systems, there are two essential methods: input-to-state stability (ISS) and sliding mode control (SMC). The ISS technique developed by Sontag (see [14, 15, 16]) is used to analyze the robustness of nonlinear 
systems and design a robust controller based on Control Lyapunov Functions. In recent years, robust control of hybrid systems based on the ISS technique has attracted attention, for instance see [17, 18, 19]. However, ISS based controllers tend to maintain system errors in a sufficiently small neighborhood of the origin, and thus have a non-zero tracking error. In contrast, sliding mode control can deal with a wide range of uncertainties and drive the system to follow a given sliding surface, thereby driving outputs to desired values without any tracking errors (see [20, 21, 22]). However, the primary disadvantage of SMC is the chattering phenomenon caused by discrete switching for keeping the system attracted to the sliding surface.

As a result, with robust control problems for bipedal systems that require high quality tracking, guarantees on rate of convergence, smoothness of control inputs, and energy efficiency, the application of ISS and SMC seem to have some inherent limitations. There is some work on robust control with application to bipedal robots based on Sliding Mode Control [23, 24], and other methods [25], [26], nevertheless these approaches just deal with a small level of uncertainty. In [27], CLF-QPs are used to adapt to parameter uncertainty in a bipedal robot, however the controller assumes full actuation, requires measurement of acceleration, and appears to only work for tracking a static pose of the robot.

The main contribution of this paper is a new Optimal Robust Control strategy that can guarantee the exponential stability of a periodic orbit for a hybrid, nonlinear, under-actuated bipedal system with significant uncertainty in the model. We assume the uncertainty is bounded and incorporate the uncertainty bounds as additional constraints of a CLF-QP. This results in the control inputs being optimal and guaranteeing the best robustness of the closed-loop system. Our method enables dealing with severe uncertainties, while also providing a guarantee on the exponential stability of the periodic orbit for the hybrid system, see Figure 1. The Lipschitz Continuity of the control inputs is guaranteed through results in [28].

The rest of the paper is organized as follows. Section II revists rapidly exponentially stabilizing control Lyapunov functions (RES-CLFs), and control Lyapunov function-based quadratic programs (CLF-QPs). Section [III discusses the adverse effects of uncertainty in the dynamics on the CLF-QP controllers. Section IV presents the proposed CLF-QP based optimal robust controller. Section VI presents simulations of the controller on a perturbed model of RABBIT, a five-link planar bipedal robot. Finally, Section VII provides concluding remarks.

\section{RAPidLy EXPONENTIALly STABILIZING CONTROL LYAPUNOV FUNCTIONS AND QUADRATIC PROGRAMS REVISITED}

In this section we start by introducing a hybrid dynamical model that captures the dynamics of a bipedal robot. We then review recent innovations on control Lyapunov functions for hybrid systems and control Lyapunov function based quadratic programs, introduced in [5] and [6] respectively.

\section{A. Model}

This paper will focus on the specific problem of walking of bipedal robots such as RABBIT (described in [29]), which is characterized by single-support continuous-time dynamics, when one foot is assumed to be in contact with the ground, and double-support discrete-time impact dynamics, when the swing foot undergoes an instantaneous impact with the ground. Such a hybrid model is obtained as,

$$
\mathcal{H}= \begin{cases}{\left[\begin{array}{c}
\dot{q} \\
\ddot{q}
\end{array}\right]=f(q, \dot{q})+g(q, \dot{q}) u,} & \left(q^{-}, \dot{q}^{-}\right) \notin S, \\
{\left[\begin{array}{c}
q^{+} \\
\dot{q}^{+}
\end{array}\right]=\Delta\left(q^{-}, \dot{q}^{-}\right),} & \left(q^{-}, \dot{q}^{-}\right) \in S,\end{cases}
$$

where $q \in \mathscr{Q}$ is the robot's configuration variables, $u \in \mathbb{R}^{m}$ is the control inputs, representing the motor torques, $\left(q^{-}, \dot{q}^{-}\right)$ represent the state before impact and $\left(q^{+}, \dot{q}^{+}\right)$represent the state after impact, $S$ represents the switching surface when the swing leg contacts the ground, and $\Delta$ represents the discretetime impact map.

We also define output functions $y \in \mathbb{R}^{m}$ of the form

$$
y(q):=H_{0} q-y_{d}(\theta(q)),
$$

where $\theta(q)$ is a strictly monotonic function of the configuration variable $q, H_{0}$ is an appropriately-sized matrix prescribing linear combinations of state variables to be controlled, and $y_{d}(\cdot)$ prescribes the desired evolution of these quantities. (See [3] for details.) The method of Hybrid Zero Dynamics (HZD) aims to drive these output functions (and their first derivatives) to zero, thereby imposing "virtual constraints" such that the system evolves on the lower-dimensional zero dynamics manifold, given by

$$
Z=\left\{(q, \dot{q}) \in T \mathscr{Q} \mid y(q)=0, L_{f} y(q, \dot{q})=0\right\},
$$

where $L_{f}$ denotes the Lie derivative [30]. In particular, the dynamics of the system on $Z$, given by $\left.\mathcal{H}\right|_{Z}$, is the underactuated dynamics of the system and is forward-invariant. Periodic motion such as walking is then a hybrid periodic orbit $\mathscr{O}$ in the statespace with $\mathscr{O}_{Z}$ being it's restriction to $Z$. (We have $\mathscr{O}=\iota_{0}\left(\mathscr{O}_{Z}\right)$, where $\iota_{0}: Z \rightarrow T \mathscr{Q}$ is the inclusion map.) Prior results on feedback control for such underactuated hybrid systems assume stability of $\mathscr{O}_{Z}$ and design controllers that stabilize $\mathscr{O}$, see [31, 5]. These controllers are revisited next.

\section{B. Input-output linearization}

If $y(q)$ has vector relative degree 2 , then the second derivative takes the form

$$
\ddot{y}=L_{f}^{2} y(q, \dot{q})+L_{g} L_{f} y(q, \dot{q}) u .
$$

We can then apply one of the following control laws,

$$
u(q, \dot{q})=u^{*}(q, \dot{q})+\mu,
$$


or

$$
u(q, \dot{q})=u^{*}(q, \dot{q})+\left(L_{g} L_{f} y(q, \dot{q})\right)^{-1} \mu,
$$

where

$$
u^{*}(q, \dot{q}):=-\left(L_{g} L_{f} y(q, \dot{q})\right)^{-1} L_{f}^{2} y(q, \dot{q}) .
$$

Defining transverse variables $\eta=\left[\begin{array}{ll}y, & \dot{y}\end{array}\right]^{T}$, and using the IO linearization controller above with the pre-control law (6), we have,

$$
\ddot{y}=\mu \text {. }
$$

Then, with the PD control

$$
\mu=\left[\begin{array}{ll}
-\frac{1}{\varepsilon^{2}} K_{P} & -\frac{1}{\varepsilon} K_{D}
\end{array}\right] \eta,
$$

the closed-loop system will become

$$
\ddot{y}+\frac{1}{\varepsilon} K_{D} \dot{y}+\frac{1}{\varepsilon^{2}} K_{P} y=0,
$$

and will be exponentially stable if we choose $K_{P}$ and $K_{D}$ such that

$$
A=\left[\begin{array}{cc}
0 & I \\
-K_{P} & -K_{D}
\end{array}\right]
$$

is Hurwitz. The $\varepsilon$ factor is used to control the rate of convergence and is needed to ensure we converge sufficiently fast so as to account for the expansion produced by the impact map, see [32] for more details.

For the work presented here, we will use the pre-control law (6) that creates the closed-loop output dynamics (8). Therefore, the input-output linearized dynamics will become

$$
\dot{\eta}=\left[\begin{array}{c}
\dot{y} \\
\ddot{y}
\end{array}\right]=\left[\begin{array}{ll}
0 & I \\
0 & 0
\end{array}\right]\left[\begin{array}{c}
y \\
\dot{y}
\end{array}\right]+\left[\begin{array}{l}
0 \\
I
\end{array}\right] \mu .
$$

The closed-loop dynamics in terms of the transverse variables $\eta$ and the states $z \in Z$ take the form,

$$
\begin{aligned}
\dot{\eta} & =\bar{f}(\eta, z)+\bar{g}(\eta, z) \mu, \\
\dot{z} & =p(\eta, z),
\end{aligned}
$$

where $\bar{f}(\eta, z)=F \eta$ and $\bar{g}(\eta, z)=G$, with

$$
F=\left[\begin{array}{ll}
0 & I \\
0 & 0
\end{array}\right], \quad G=\left[\begin{array}{l}
0 \\
I
\end{array}\right] \text {. }
$$

\section{CLF-based control}

An alternative control approach based on control Lyapunov functions, introduced in [5], provides guarantees of rapid exponential stability for the traverse variables $\eta$. In particular, a function $V_{\varepsilon}(\eta)$ is a rapidly exponentially stabilizing control Lyapunov function (RES-CLF) for the system (13) if there exist positive constants $c_{1}, c_{2}, c_{3}>0$ such that for all $0<\varepsilon<1$ and all states $(\eta, z)$ it holds that

$$
\begin{gathered}
c_{1}\|\eta\|^{2} \leq V_{\varepsilon}(\eta) \leq \frac{c_{2}}{\varepsilon^{2}}\|\eta\|^{2}, \\
\dot{V}_{\varepsilon}(\eta, \mu)+\frac{c_{3}}{\varepsilon} V_{\varepsilon}(\eta) \leq 0 .
\end{gathered}
$$

In our problem, we chose a CLF candidate as follows

$$
V_{\varepsilon}(\eta)=\eta^{T}\left[\begin{array}{cc}
\frac{1}{\varepsilon} I & 0 \\
0 & I
\end{array}\right] P\left[\begin{array}{cc}
\frac{1}{\varepsilon} I & 0 \\
0 & I
\end{array}\right] \eta=: \eta^{T} P_{\varepsilon} \eta,
$$

where $P$ is the solution of the Lyapunov equation $A^{T} P+$ $P A=-Q$ (where $A$ is given by (11) and $Q$ is any symmetric positive-definite matrix). The time derivative of the CLF (17) is computed as

$$
\dot{V}_{\varepsilon}(\eta, \mu)=L_{\bar{f}} V_{\varepsilon}(\eta, z)+L_{\bar{g}} V_{\varepsilon}(\eta, z) \mu,
$$

where

$$
\begin{aligned}
L_{\bar{f}} V_{\varepsilon}(\eta, z) & =\eta^{T}\left(F^{T} P_{\varepsilon}+P_{\varepsilon} F\right) \eta, \\
L_{\bar{g}} V_{\varepsilon}(\eta, z) & =2 \eta^{T} P_{\varepsilon} G .
\end{aligned}
$$

We can thus construct the set of control $\mu$ that satisfies the RES condition (16) as follows: We define the set,

$$
K_{\varepsilon}(\eta, z)=\left\{\mu_{\varepsilon} \in U: \psi_{0, \varepsilon}(\eta, z)+\psi_{1, \varepsilon}(\eta, z) \mu_{\varepsilon} \leq 0\right\},
$$

where,

$$
\begin{aligned}
& \psi_{0, \varepsilon}(\eta, z)=L_{\bar{f}} V_{\varepsilon}(\eta, z)+\frac{c_{3}}{\varepsilon} V_{\varepsilon}(\eta, z) \\
& \psi_{1, \varepsilon}(\eta, z)=L_{\bar{g}} V_{\varepsilon}(\eta, z) .
\end{aligned}
$$

Then, it can be show that for any Lipschitz continuous feedback control law $\mu_{\varepsilon}(\eta, z) \in K_{\varepsilon}(\eta, z)$, it holds that

$$
\|\eta(t)\| \leq \frac{1}{\varepsilon} \sqrt{\frac{c_{2}}{c_{1}}} e^{-\frac{c_{3}}{2 \varepsilon} t}\|\eta(0)\|,
$$

i.e. the rate of exponential convergence to the zero dynamics manifold can be directly controlled with the constant $\varepsilon$ through $\frac{c_{3}}{\varepsilon}$. One such controller is the pointwise min-norm control law [33], formulated as,

Pointwise min-norm:

$$
\mu_{\varepsilon}(\eta, z)=\left\{\begin{array}{lll}
-\frac{\psi_{0, \varepsilon}(\eta, z) \psi_{1, \varepsilon}(\eta, z)}{\psi_{1, \varepsilon}(\eta, z)^{T} \psi_{1, \varepsilon}(\eta, z)} & \text { if } & \psi_{0, \varepsilon}(\eta, z)>0 \\
0 & \text { if } & \psi_{0, \varepsilon}(\eta, z) \leq 0
\end{array}\right\}
$$

\section{CLF-based Quadratic Programs}

CLF-based quadratic programs were introduced in [6], where $\mu_{\varepsilon}$ was directly selected through an online quadratic program to meet 16 :

\section{CLF-QP:}

$$
\begin{array}{ll}
\underset{\mu}{\operatorname{argmin}} & \mu^{T} \mu \\
\text { s.t. } & \psi_{0, \varepsilon}(\eta, z)+\psi_{1, \varepsilon}(\eta, z) \mu \leq 0 .
\end{array}
$$

The quadratic program formulation now lets us incorporate additional constraints of the form $A(q, \dot{q}) \mu \leq b(q, \dot{q})$ into the control problem. However, for these additional constraints to be satisfied and to ensure feasibility of the quadratic program, 
we relax the RES-CLF bound on the time-derivative of $V$. We do this by requiring $\dot{V}_{\varepsilon}(\eta) \leq-c_{3} / \varepsilon V_{\varepsilon}(\eta)+d_{1}$, where $d_{1}$ is typically a small positive quantity. The relaxed CLF-QP is then written as

\section{Relaxed CLF-QP:}

$$
\begin{array}{ll}
\underset{\mu, d_{1}}{\operatorname{argmin}} & \mu^{T} \mu+p_{1} d_{1}^{2} \\
\text { s.t. } & \psi_{0, \varepsilon}(\eta, z)+\psi_{1, \varepsilon}(\eta, z) \mu \leq d_{1}, \\
& A(q, \dot{q}) \mu \leq b(q, \dot{q}),
\end{array}
$$

where $p_{1}$ is a large positive number that represents the penalty of relaxing the inequality. The formulation in 24) deals with additional state-based inequality constraint $A(q, \dot{q}) \mu \leq$ $b(q, \dot{q})$, that potentially cannot ensure the same type of stability claims as those provided by [5, Thm. 2], as the relaxations in the bound on the time-derivative of $V_{\varepsilon}$ result in a loss of the RES-CLF quality for $V_{\varepsilon}$. However, as we will see in this paper, under appropriate conditions, we still retain the stability of the hybrid periodic orbit. We will demonstrate this on the torque saturated controller:

\section{CLF-QP with Torque Saturation:}

$$
\begin{array}{ll}
\underset{\mu, d_{1}}{\operatorname{argmin}} & \mu^{T} \mu+p_{1} d_{1}^{2} \\
\text { s.t. } & \psi_{0, \varepsilon}(\eta, z)+\psi_{1, \varepsilon}(\eta, z) \mu \leq d_{1}, \\
& \left(L_{g} L_{f} y(q, \dot{q})\right)^{-1} \mu \geq\left(u_{\min }-u^{*}\right), \\
& \left(L_{g} L_{f} y(q, \dot{q})\right)^{-1} \mu \leq\left(u_{\max }-u^{*}\right) .
\end{array}
$$

Having presented recent developments in control Lyapunov functions and control Lyapunov functions based quadratic programs for hybrid dynamical systems, we next consider the effect of uncertainty in the dynamics on these controllers.

\section{ADVERSE EFFECTS OF UNCERTAINTY IN DYNAMICS ON THE CLF-QP CONTROLLER}

The CLF-based approaches presented in Section [I] have several interesting properties. Firstly, they provide a guarantee on the exponential stability of the hybrid system, they are optimal with respect to some cost function, result in the minimum control effort, and provide means of balancing conflicting requirements between performance and state-based constraints. These controllers were even successfully implemented on MABEL, see [5, 6]. However, a primary disadvantage of these controllers is that they require an accurate dynamical model of the system. Specifically, as we will see, even for a simpler bipedal model such as RABBIT (compared to MABEL), uncertainty in mass and inertia properties of the model can cause bad control quality leading to tracking errors, and could potentially lead to walking that is unstable.

If we consider uncertainty in the dynamics and assume that the functions, $f(q, \dot{q}), g(q, \dot{q})$ of the real dynamics (1), are unknown, we then have to design our controller based on nominal functions $\tilde{f}(q, \dot{q}), \tilde{g}(q, \dot{q})$. Thus, the pre-control law
(6) is reformulated as

$$
u(q, \dot{q})=u^{*}(q, \dot{q})+\left(L_{\tilde{g}} L_{\tilde{f}} y(q, \dot{q})\right)^{-1} \mu,
$$

with

$$
u^{*}(q, \dot{q}):=-\left(L_{\tilde{g}} L_{\tilde{f}} y(q, \dot{q})\right)^{-1} L_{\tilde{f}}^{2} y(q, \dot{q}) .
$$

Substituting $u(q, \dot{q})$ from (26) into (4), the second derivative of the output, $y(q)$, then becomes

$$
\ddot{y}=\mu+\Delta_{1}+\Delta_{2} \mu,
$$

where

$$
\begin{aligned}
& \Delta_{1}=L_{f}^{2} y(q, \dot{q})-L_{g} L_{f} y(q, \dot{q})\left(L_{\tilde{g}} L_{\tilde{f}} y(q, \dot{q})\right)^{-1} L_{\tilde{f}}^{2} y(q, \dot{q}), \\
& \Delta_{2}=L_{g} L_{f} y(q, \dot{q})\left(L_{\tilde{g}} L_{\tilde{f}} y(q, \dot{q})\right)^{-1}-I .
\end{aligned}
$$

Using $F$ and $G$ as in (14) and defining,

$$
\Delta H=\left[\begin{array}{c}
0 \\
\Delta_{1}
\end{array}\right], \quad \Delta G=\left[\begin{array}{c}
0 \\
\Delta_{2}
\end{array}\right],
$$

the closed-loop system now takes the form

$$
\dot{\eta}=F \eta+(G+\Delta G) \mu+\Delta H .
$$

For the following sections, we will abuse notation and redefine $\bar{f}=F \eta+\Delta H, \bar{g}=G+\Delta G$.

Clearly for $\Delta H \neq 0$, or $\Delta G \neq 0$, the PD control 9 does not stabilize the system dynamics. In fact for $\Delta H \neq 0$, the closed-loop system does not have an equilibrium, and for $\Delta G \neq 0$, the controller could potentially destabilize the system. This raises the question of whether it's possible for controllers to account for this model uncertainty, and if so, how do we design such a controller.

\section{CLF based Quadratic Programs with Robust CONTROL}

Having discussed the effect of model uncertainty on the control Lyapunov function based controllers, we now develop a controller that can guarantee tracking and stability in the presence of bounded uncertainty. As we will see, both stability and tracking performance (rate of convergence and errors going to zero) are still retained for all uncertainty within a particular bound. This is evidenced in Figure 3 . For uncertainty that exceeds the specified bound, there is graceful degradation in performance.

\section{A. Proposed Robust CLF-QP Controller}

We start with the closed-loop model with uncertainty as developed in (31) and develop the robust controller as follows. With the CLF defined in (17), we have:

$$
\dot{V}_{\varepsilon}=L_{\bar{f}} V_{\varepsilon}(\eta, z)+L_{\bar{g}} V_{\varepsilon}(\eta, z) \mu,
$$

where we have

$$
\begin{aligned}
& L_{\bar{f}} V_{\varepsilon}(\eta, z)=\eta^{T}\left(F^{T} P_{\varepsilon}+P_{\varepsilon} F\right) \eta+2 \eta^{T} P_{\varepsilon} \Delta H, \\
& L_{\bar{g}} V_{\varepsilon}(\eta, z)=2 \eta^{T} P_{\varepsilon}(G+\Delta G) .
\end{aligned}
$$

Then, in order to guarantee the standard RES condition (16), we will have to guarantee:

$$
\tilde{\Psi}_{0, \varepsilon}+\tilde{\Psi}_{1, \varepsilon} \mu \leq 0
$$


where

$$
\begin{aligned}
& \tilde{\Psi}_{0, \varepsilon}=\eta^{T}\left(F^{T} P_{\varepsilon}+P_{\varepsilon} F\right) \eta+2 \eta^{T} P_{\varepsilon} \Delta H+\frac{c_{3}}{\varepsilon} V_{\varepsilon}, \\
& \tilde{\Psi}_{1, \varepsilon}=2 \eta^{T} P_{\varepsilon}(G+\Delta G) .
\end{aligned}
$$

In general, we can not satisfy the inequality (34) for all possible unknown $\Delta H, \Delta G$ in 35. To address this, we assume the uncertainty is bounded as follows

$$
\|\Delta H\| \leq \Delta H_{\max }, \quad\|\Delta G\| \leq \Delta G_{\max }
$$

where the first norm is a vector norm, while the second norm is a matrix norm.

The goal of the robust control design is then to find the control $\mu$ satisfying the RES condition (34), evaluated through the given bounds of uncertainty in 36. Specifically, with the assumption on bounds on the uncertainty, the RES condition (34) will hold if the following inequalities holds

$$
\begin{array}{r}
\tilde{\Psi}_{0, \varepsilon}^{\max }+\tilde{\Psi}_{1, \varepsilon}^{p} \mu \leq 0 \\
\tilde{\Psi}_{0, \varepsilon}^{\max }+\tilde{\Psi}_{1, \varepsilon}^{n} \mu \leq 0,
\end{array}
$$

where

$$
\begin{aligned}
\tilde{\Psi}_{0, \varepsilon}^{\max } & =\max \left(\tilde{\Psi}_{0, \varepsilon}^{n}, \tilde{\Psi}_{0, \varepsilon}^{p}\right), \\
\tilde{\Psi}_{0, \varepsilon}^{p} & =\eta^{T}\left(F^{T} P_{\varepsilon}+P_{\varepsilon} F\right) \eta+2 \eta^{T} P_{\varepsilon} \Delta H_{\text {max }}+\frac{c_{3}}{\varepsilon} V_{\varepsilon}, \\
\tilde{\Psi}_{0, \varepsilon}^{n} & =\eta^{T}\left(F^{T} P_{\varepsilon}+P_{\varepsilon} F\right) \eta-2 \eta^{T} P_{\varepsilon} \Delta H_{\max }+\frac{c_{3}}{\varepsilon} V_{\varepsilon}, \\
\tilde{\Psi}_{1, \varepsilon}^{p} & =2 \eta^{T} P_{\varepsilon}\left(G+\Delta G_{\text {max }}\right), \\
\tilde{\Psi}_{1, \varepsilon}^{n} & =2 \eta^{T} P_{\varepsilon}\left(G-\Delta G_{\text {max }}\right) .
\end{aligned}
$$

We can then incorporate these inequalities into a new relaxed CLF-QP as follows:

\section{Robust CLF-QP:}

$$
\begin{array}{ll}
\underset{\mu, d_{1}, d_{2}}{\operatorname{argmin}} & \mu^{T} \mu+p_{1} d_{1}^{2}+p_{2} d_{2}^{2} \\
\text { s.t. } & \tilde{\psi}_{0, \varepsilon}^{\max }(\eta, z)+\tilde{\psi}_{1, \varepsilon}^{p}(\eta, z) \mu \leq d_{1}, \\
& \tilde{\psi}_{0, \varepsilon}^{\max }(\eta, z)+\tilde{\psi}_{1, \varepsilon}^{n}(\eta, z) \mu \leq d_{2}, \\
& \left(L_{\tilde{g}} L_{\tilde{f}} y(q, \dot{q})\right)^{-1} \mu \geq\left(u_{\min }-u^{*}\right), \\
& \left(L_{\tilde{g}} L_{\tilde{f}} y(q, \dot{q})\right)^{-1} \mu \leq\left(u_{\max }-u^{*}\right) .
\end{array}
$$

Here $d_{1}, d_{2}$ are the relaxations of the inequalities in (37). The relaxed inequality has the benefit of making the solution to the above Quadratic program feasible and Lipschitz continuous in a larger region (see [28]). The above robust CLF-QP enables the controller to be robust to all model perturbations that are within the specified bound in (36). In particular, both stability and tracking performance (rate of convergence and errors going to zero) are still retained for all uncertainty within bounds. However, since we are expressing this as a relaxed CLF-QP, we lose the strict RES condition and thus require additional formulations to formally guarantee stability. We will develop these formulations next.

\section{SUFFICIENT CONDITIONS FOR THE STABILITY OF CLF WITH RELAXED INEQUALITY}

As we saw in the previous section, we can formulate a relaxed CLF-QP to be able to incorporate additional constraints while allowing for violation of the RES-CLF condition. This could lead to potential instability. However, here, we establish sufficient conditions under which we still retain the exponential stability of the hybrid periodic orbit. We will then use this result to establish stability of the robust CLF-QP.

\section{A. Stability of the Relaxed CLF-QP Controller}

The standard RES-CLF will guarantee the following inequality:

$$
\dot{V}_{\varepsilon}+\frac{c_{3}}{\varepsilon} V_{\varepsilon} \leq 0
$$

The CLF-QP with the relaxed inequality will take the form

$$
\dot{V}_{\varepsilon}+\frac{c_{3}}{\varepsilon} V_{\varepsilon} \leq d_{1} .
$$

We define:

$$
d_{\varepsilon}(t)=\dot{V}_{\varepsilon}+\frac{c_{3}}{\varepsilon} V_{\varepsilon}
$$

and

$$
w_{\varepsilon}(t)=\int_{0}^{t} \frac{d_{\varepsilon}(\tau)}{V_{\varepsilon}} \mathrm{d} \tau
$$

Remark 1: First we define $T_{I}^{\varepsilon}(\eta, z)$ to be the time-toimpact, that signifies ending time of the step. Then, intuitively, $w_{\varepsilon}\left(T_{I}^{\varepsilon}(\eta, z)\right)$ indicates a scaled version of the total violation of the RES-CLF bound in (40) over one complete step. If $w_{\varepsilon}\left(T_{I}^{\varepsilon}(\eta, z)\right) \leq 0$, it implies that $V_{\varepsilon}\left(T_{I}^{\varepsilon}(\eta, z)\right)$ is less than or equal to what would have resulted if the RES-CLF bound had not been violated at all. As we will see in the following theorem, we will in fact only require $w_{\varepsilon}\left(T_{I}^{\varepsilon}(\eta, z)\right)$ to be upper bounded by a positive constant for exponential stability.

We then have the following theorem:

Theorem 1: Let $\mathscr{O}_{Z}$ be an exponentially stable periodic orbit of the hybrid zero dynamics $\left.\mathscr{H}\right|_{Z}$ transverse to $S \cap Z$ and the continuous dynamics (1) of $\mathscr{H}$ controlled by a CLF$Q P$ with relaxed inequality (24). Then there exists an $\bar{\varepsilon}>0$ and an $\bar{w}_{\varepsilon} \geq 0$ such that for each $0<\varepsilon<\bar{\varepsilon}$, if the solution $\mu_{\varepsilon}(\eta, z)$ of the CLF-QP (24) satisfies $w_{\varepsilon}\left(T_{I}^{\varepsilon}(\eta, z)\right) \leq \bar{w}_{\varepsilon}$, then $\mathscr{O}=\iota_{0}\left(\mathscr{O}_{Z}\right)$ is an exponentially stable hybrid periodic orbit of $\mathscr{H}$.

\section{Proof: See [34].}

\section{B. Stability of the Robust CLF-QP Controller}

It is clear that if there is no uncertainty in the system, i.e., $\Delta H_{\max }=\Delta G_{\max }=0$, then the robust CLF-QP (39) is exactly the same as the CLF-QP with torque saturation (25). In contrast, if uncertainties are too significant, say $\Delta H_{\max } \rightarrow$ $\infty, \Delta G_{\max } \rightarrow \infty$, it is obvious that we cannot guarantee the stability of the hybrid system. We require the uncertainty to be bounded. Moreover, model uncertainty further complicates the problem, since it causes the periodic orbit to move from $\mathscr{O}_{Z}$ to $\overline{\mathscr{O}}_{Z}$, corresponding to a periodic orbit for the new perturbed model. To establish stability guarantees, we restate Theorem 1 for the CLF-QP with robust control as follows: 


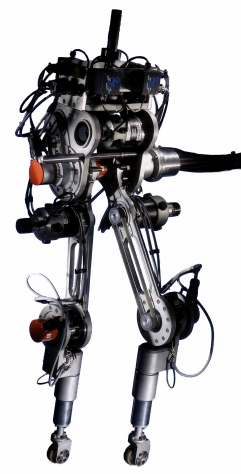

(a) RABBIT

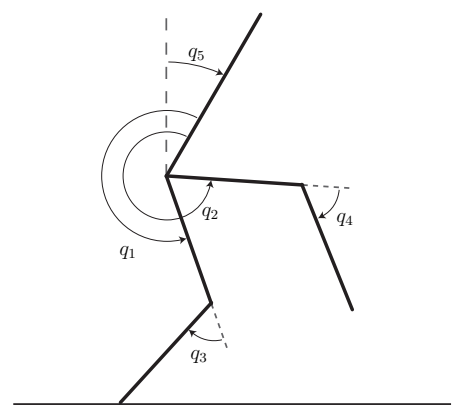

(b) Coordinate system
Fig. 2: (a) RABBIT, a planar five-link bipedal robot with nonlinear, hybrid and underactuated dynamics. (b) The the associated generalized coordinate system used, where $q_{1}, q_{2}$ are the relative stance and swing leg femur angles referenced to the torso, $q_{3}, q_{4}$ are the relative stance and swing leg knee angles, and $q_{5}$ is the absolute torso angle in the world frame.

Theorem 2: Let $\overline{\mathscr{O}}_{Z}$ be an exponentially stable periodic orbit of the hybrid zero dynamics $\left.\mathscr{H}\right|_{Z}$ transverse to $S \cap Z$ and the continuous dynamics (1) of $\mathscr{H}$ controlled by the robust CLF-QP (39). Then there exists an $\bar{\varepsilon}>0 ; u_{\text {min }}^{\varepsilon}>$ $u_{\min }^{K_{\varepsilon}}, u_{\max }^{\varepsilon}<u_{\max }^{K_{\varepsilon}}$ and $\Delta G_{\text {max }}^{\varepsilon}, \Delta H_{\text {max }}^{\varepsilon}$, such that for each $0<\varepsilon<\bar{\varepsilon}$, if

$$
\begin{aligned}
\left\|\Delta G_{\max }\right\| & \leq\left\|\Delta G_{\text {max }}^{\varepsilon}\right\| \\
\left\|\Delta H_{\text {max }}\right\| & \leq\left\|\Delta H_{\text {max }}^{\varepsilon}\right\| \\
u_{\min } & \leq u_{\text {min }}^{\varepsilon} \\
u_{\max } & \geq u_{\text {max }}^{\varepsilon},
\end{aligned}
$$

the CLF-QP with robust control (39) will imply that $\overline{\mathscr{O}}=$ $\iota_{0}\left(\overline{\mathscr{O}}_{Z}\right)$ is an exponentially stable hybrid periodic orbit of $\mathscr{H}$.

Proof: Follows from $(39)$ and Theorem 1.

\section{Simulation of Robust Control with TORQue SATURATION}

To demonstrate the effectiveness of the proposed robust CLF-QP controller, we will conduct numerical simulations on the model of RABBIT (shown in Figure 2, a planar fivelink bipedal robot with a torso and two legs with revolute knees that terminate in point feet. RABBIT weighs $32 \mathrm{~kg}$, has four brushless DC actuators with harmonic drives to control the hip and knee angles, and is connected to a rotating boom which constrains the robot to walk in a circle, approximating planar motion in the sagittal plane. Further description of RABBIT and the associated mathematical model can be found in [29, 35]. Fundamental issues in dynamic walking and running on RABBIT can be found in [35] and [36].

For RABBIT, the stance phase is parametrized by a suitable set of coordinates, given by $q:=\left(q_{1}, q_{2}, q_{3}, q_{4}, q_{5}\right)$ and as illustrated in Fig 2 . Here, $q_{1}$ and $q_{2}$ are the femur angles (referenced to the torso), $q_{3}$ and $q_{4}$ are the knee angles, and $q_{5}$ is the absolute angle of the torso. Because RABBIT has point feet (while many other legged robots have flat feet), the stance

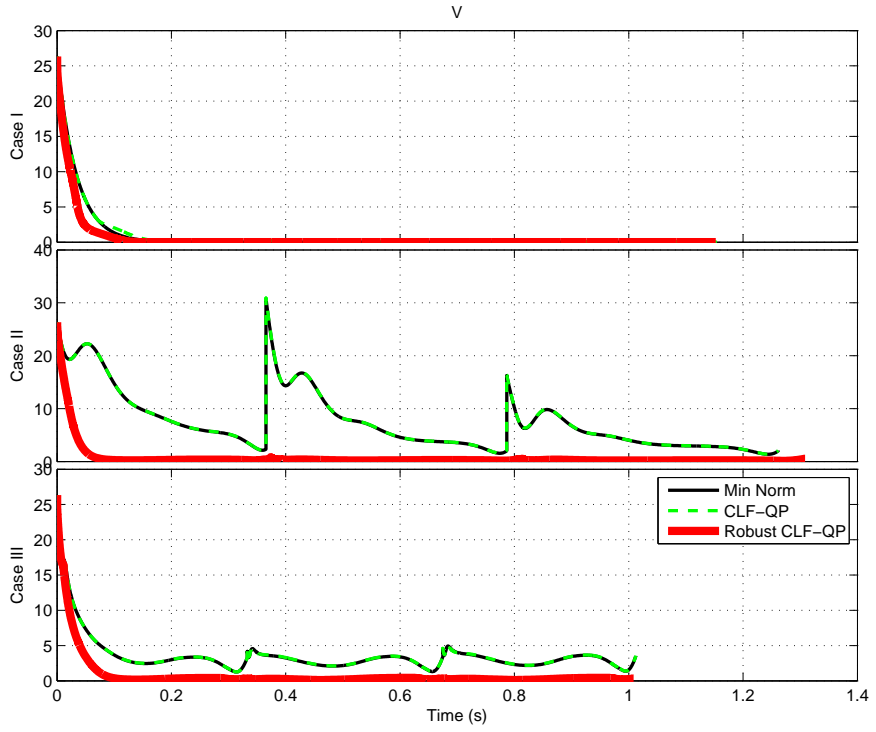

Fig. 3: Control Lyapunov function for the three controllers: Min Norm, CLF-QP, Robust CLF-QP, for the cases I-III of model perturbations. Notice that the convergence performance (both rate of convergence and tracking error being driven to zero) of the proposed robust CLF-QP controller remains the same across the different model perturbations.

phase dynamics are underactuated with the system possessing 4 actuated degrees-of-freedom (DOF) and 1 underactuated DOF.

For the purpose of evaluating the robust CLF-QP controller, we will consider a periodic walking gait, with step speed of $0.9 \mathrm{~m} / \mathrm{s}$ and step length of $0.45 \mathrm{~m}$, that is developed for a nominal model of RABBIT. The controller is also developed for this nominal model. The simulation is then carried out on a perturbed model of RABBIT, where the perturbation is introduced by scaling all mass and inertia parameters of each link by a fixed constant scale factor. The perturbed model is unknown to the controller and will serve as an uncertainty injected into the model. We will illustrate four separate cases of scaling the mass and inertia:

$$
\begin{aligned}
\text { Case I } & : \text { model scale }=1 \\
\text { Case II } & : \text { model scale }=1.5 \\
\text { Case III } & : \text { model scale }=0.7 \\
\text { Case IV } & : \quad \text { model scale }=3 ;
\end{aligned}
$$

For each case, we will compare the quality of three controllers

Controller A: Min-norm Controller (22)

Controller B : CLF-QP torque saturation controller 25)

Controller $C$ : CLF-QP robust controller (39).

Note that controllers $\mathrm{B}, \mathrm{C}$ enable limiting the torque to be within a user specified bound. For a fair comparison, we set these torque bounds slightly below the maximum torque that controller A uses for each particular case of model perturbation. In particular, the torque saturations were set as 


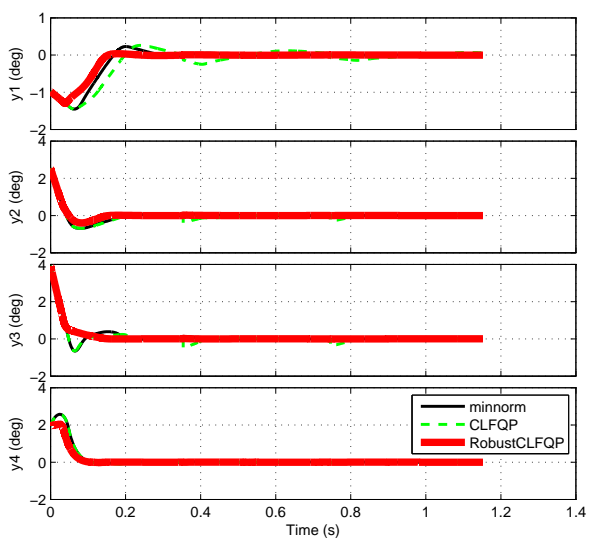

(a) Case I: model scale $=1$

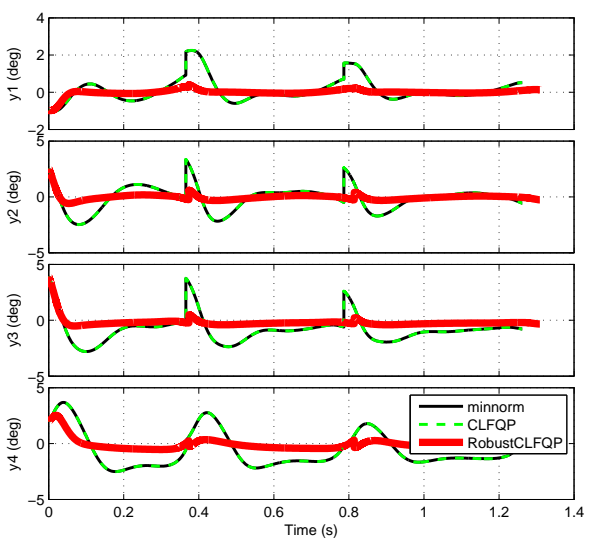

(b) Case II: model scale $=1.5$

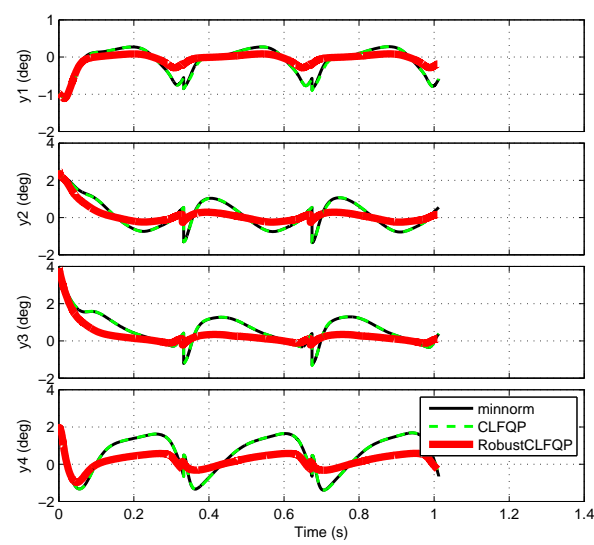

(c) Case III: model scale $=0.7$

Fig. 4: Tracking errors of the virtual constraints (2) based on the simulation of cases I-III of perturbed model of RABBIT with three controllers as described in Section $\mathrm{VI}$. Simulation of three walking steps are shown.

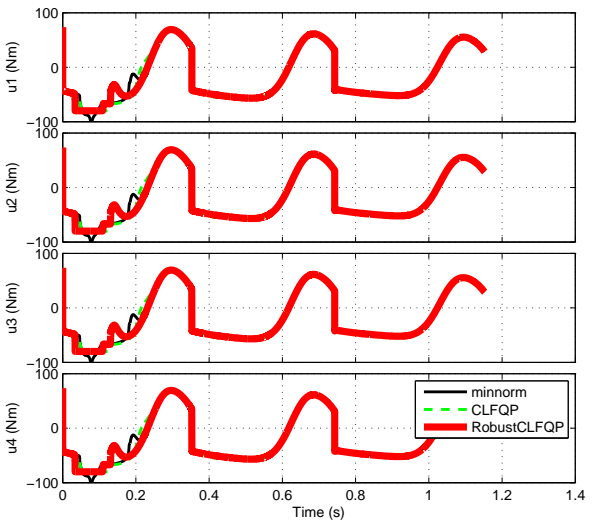

(a) Case I: model scale $=1$

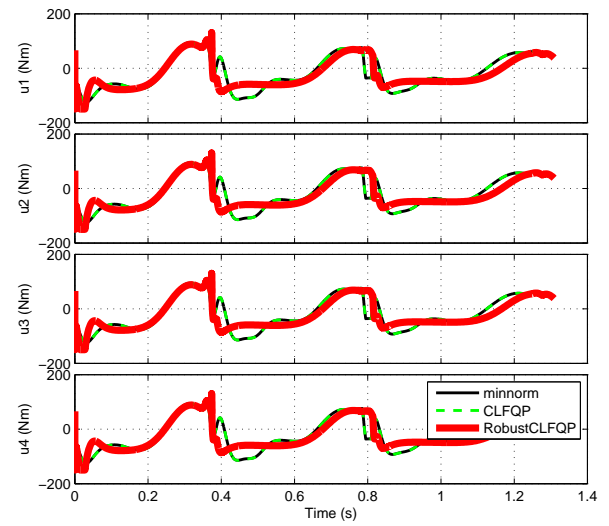

(b) Case II: model scale $=1.5$

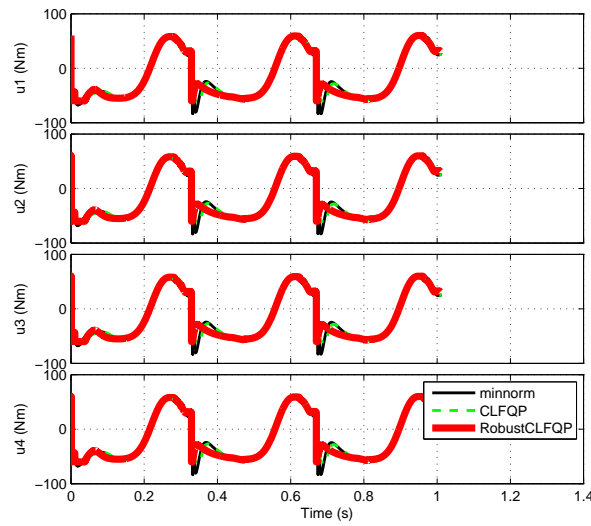

(c) Case III: model scale $=0.7$

Fig. 5: Control inputs (motor torques for stance and swing legs) based on the simulation of cases I-III of perturbed model of RABBIT with three controllers as described in Section VI. Simulation of three walking steps are shown.

follows: $60 \mathrm{Nm}, 80 \mathrm{Nm}, 150 \mathrm{Nm}$, and $300 \mathrm{Nm}$ for the model scale equals to $0.7,1,1.5,3$ respectively.

Each of the four models are evaluated with the three sets of controllers. The results are presented in Figures 3, 4, 5, 7.

Remark 2: From Figure 3 , note that the performance (both rate of convergence and driving the tracking error to zero) of the proposed robust CLF-QP controller remains same for all the considered model variations.

Remark 3: For Case I: model scale $=1$, when there is no uncertainty, we can observe from the top plot of Figure 3 and from Figure 4a that the robust CLF-QP controller (controller C) performs better than the two other controllers. This is because the robust controller is trying to satisfy the convergence bound on the worst possible model perturbation. Also notice that the CLF-QP with torque saturation (controller B) does slightly worse than the min-norm controller (controller A). This is due to the torque saturation that is slightly below the maximum torque the min-norm controller uses.

For Case II: model scale $=1.5$ and Case III: model scale $=0.7$, where mass and inertia were increased by $50 \%$ and reduced by $30 \%$ respectively, we can observe from the track- ing error plots in Figures $4 \mathrm{~b}$, 4c, that while the min-norm controller (controller A) and the CLF-QP torque saturation controller (controller $\mathrm{B}$ ) have non-zero tracking errors during each step, the robust CLF-QP controller (controller C) still performs perfectly, with tracking errors converging to zero. Thus, even with this level of uncertainty, the proposed robust CLFQP controller is able to maintain the same control performance quality as in Case I (no model uncertainty). This is especially evident in Figure 3, where we clearly see that the robust CLFQP maintains the same rate of convergence for all model perturbations. Note that these comparisons are fair between the controllers since we maintain the same range of control inputs for each case of model perturbation, as evidenced in the control input plots in Figures 5

In Case IV: model scale $=3$, where we set up a highly significant level of uncertainty by scaling all mass and inertia properties by a factor of 3 , while the Controllers A and $\mathrm{B}$ fail to sustain walking, the proposed CLF-QP controller is still able to regulate the outputs with only a slight degradation, see Figure 6 However, it must be noted that the underlying periodic orbit $\mathscr{O}_{Z}$ is unstable, and the walking slows down after 


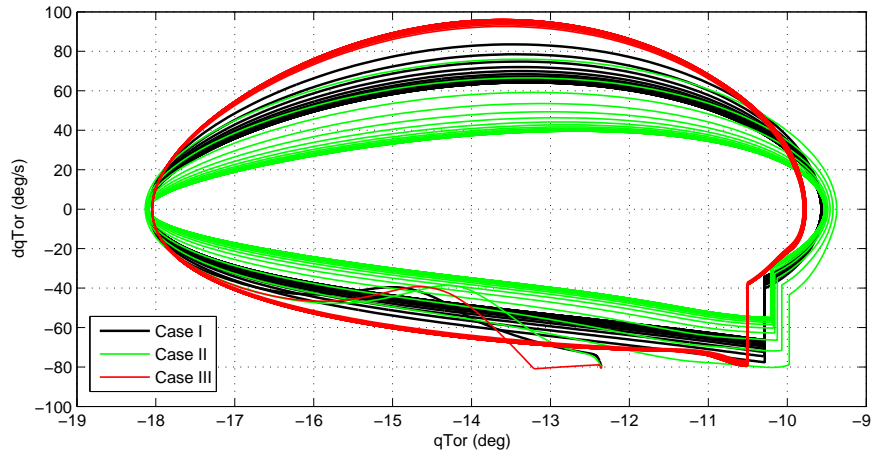

Fig. 7: Phase portrait of the torso angle for walking simulation in 25 steps for Cases I-III with the proposed CLF-QP robust controller, (39). As is evident, due to the different levels of uncertainty in the model, the walking settles to three different periodic orbits for each of the three cases respectively. Also notice that for a heavier robot (Case II with mass scale $=1.5$ ), the velocities are slower.

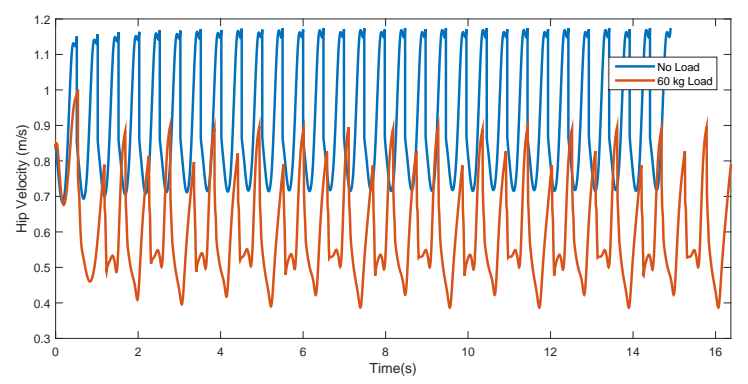

Fig. 8: The hip velocity of bipedal robot for two cases: No load and carrying heavy load of $60 \mathrm{~kg}$ ( $188 \%$ of the body mass) on the torso. Simulation of 30 steps is shown. From the plot, we can see that while the average walking speed in the nominal no load case is $0.9 \mathrm{~m} / \mathrm{s}$, for the $60 \mathrm{~kg}$ load the walking speed is slower at $0.65 \mathrm{~m} / \mathrm{s}$ and it has a 2-step periodic solution.

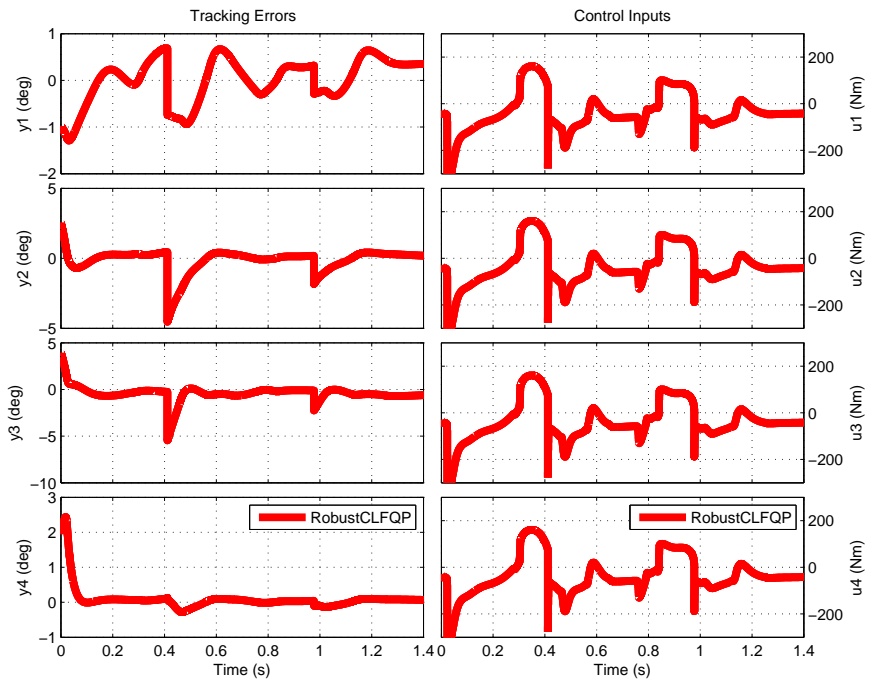

Fig. 6: Tracking errors and control outputs for Case IV: model scale $=3$.

several steps to a complete stop. Nonetheless, the controller is able to perform its task with such a large model perturbation. Future research will be directed towards addressing what can potentially be done for handling the effect of uncertainty for the underactuated dynamics.

Figure 7 illustrates the simulation of 25 steps of the proposed CLF-QP based robust controller for three cases of uncertainty as specified in Case I-III. This illustrates the controller converging to three periodic orbits that slightly vary from each other. As mentioned, the uncertainty in the model causes the underlying periodic orbit to change.

Figure 8 illustrates the hip velocity while carrying an unknown $60 \mathrm{~kg}$ load on the torso, corresponding to an additional $188 \%$ mass. Figure 1 illustrates walking with the carried unknown load randomly varying at each step.

Although the proposed robust CLF-QP based controller appears impressive, a primary limitation of the controller is that it always assumes the worst-case scenario with maximum model perturbation within the specified bounds, even when the model is known perfectly and there is no uncertainty. This results in the controller being unnecessarily aggressive, and may cause problems in experiments.

\section{CONCLUSION}

In summary, we have presented a novel optimal robust control method that successfully handles significantly high model uncertainty (up to $200 \%$ increase in mass and inertia) and respecting torque saturations, while still retaining stability of walking for a nonlinear, hybrid, underactuated, five-link planar bipedal robot. The proposed robust controller is formulated based on recent advances in rapidly exponentially stabilizing control Lyapunov functions for hybrid systems, and control Lyapunov function based quadratic programs. Future directions involve experimental validations on an underactuated bipedal robot and developing controllers that can estimate the uncertainty in the model.

\section{REFERENCES}

[1] E. Westervelt, J. Grizzle, and D. Koditschek, "Hybrid zero dynamics of planar biped walkers," IEEE Transactions on Automatic Control, vol. 48, no. 1, pp. 42-56, January 2003.

[2] E. R. Westervelt, J. W. Grizzle, C. Chevallereau, J. H. Choi, and B. Morris, Feedback Control of Dynamic Bipedal Robot Locomotion. Boca Raton: CRC Press, 2007.

[3] K. Sreenath, H. Park, I. Poulakakis, and J. Grizzle, "A compliant hybrid zero dynamics controller for stable, efficient and fast bipedal walking on MABEL," IJRR, vol. 30, pp. 1170-1193, 2011.

[4] K. Sreenath, H.-W. Park, I. Poulakakis, and J. W. Grizzle, "Embedding active force control within the compliant hybrid zero dynamics to achieve stable, fast running on MABEL," The International Journal of Robotics Research (IJRR), vol. 32, no. 3, pp. 324-345, March 2013. 
[5] A. D. Ames, K. Galloway, J. W. Grizzle, and K. Sreenath, "Rapidly Exponentially Stabilizing Control Lyapunov Functions and Hybrid Zero Dynamics," IEEE Trans. Automatic Control, 2014.

[6] K. Galloway, K. Sreenath, A. D. Ames, and J. W. Grizzle, "Torque saturation in bipedal robotic walking through control lyapunov function based quadratic programs," IEEE Access, to appear, 2015.

[7] A. D. Ames and M. Powell, "Towards the unification of locomotion and manipulation through control lyapunov functions and quadratic programs," in Control of CyberPhysical Systems, Lecture Notes in Control and Information Sciences, vol. 449, 2013, pp. 219-240.

[8] B. Morris, M. J. Powell, and A. D. Ames, "Sufficient conditions for the lipschitz continuity of qp-based multiobjective control of humanoid robots," in IEEE Conference on Decision and Control, Florence, Italy, December 2013, pp. 2920-2926.

[9] F. L. Lewis, D. Vrabie, and V. L. Syrmos, Optimal Control. John Wiley \& Sons, 2012.

[10] K. Zhou, J. Doyle, and K. Glover, Robust and Optimal Control. Prentice Hall PTR, 1996.

[11] F. Lin, Robust Control Design: An Optimal Control Approach. John Wiley \& Sons, 2007.

[12] H. T. dn S. Shu and F. Lin, "An optimal control approach to robust tracking of linear systems," International Journal of Control, vol. 82, pp. 525-540, 2009.

[13] F. Lin, "An optimal control approach to robust control design," International Journal of Control, vol. 73, pp. 177-186, 2000.

[14] E. D. Sontag, "On the input-to-state stability property," European Journal of Control, vol. 1, pp. 24-36, 1995.

[15] E. D. Sontag and Y. Wang, "On characterizations of the input-to-state stability property," Systems \& Control Letters, vol. 24, pp. 351-359, 1995.

[16] D. Angeli, E. Sontag, and Y. Wang, "A characterization of integral input-to-state stability," IEEE Transactions on Automatic Control, vol. 45, pp. 1082-1097, 2000.

[17] L. Vu, D. Chatterjee, and D. Liberzon, "Input-to-state stability of switched systems and switching adaptive control," Automatica, vol. 43, pp. 639-646, 2007.

[18] J. P. Hespanhaa, D. Liberzon, and A. R. Teel, "Lyapunov conditions for input-to-state stability of impulsive systems," Automatica, vol. 44, pp. 2735-2744, 2008.

[19] C. Cai and A. R. Teel, "Characterizations of input-tostate stability for hybrid systems," Systems \& Control Letters, vol. 58, pp. 47-53, 2009.

[20] C. Edwards and S. Spurgeon, Sliding Mode Control: Theory And Applications. CRC Press, 1998.

[21] S. V. Drakunov and V. I. Utkin, "Sliding mode control in dynamic systems," International Journal of Control, vol. 55, pp. 1029-1037, 1992.

[22] M. Zhihong, A. Paplinski, and H. Wu, "A robust mimo terminal sliding mode control scheme for rigid robotic manipulators," IEEE Transactions on Automatic Control, vol. 39, pp. 2464-2469, 1994.
[23] S. G. Tzafestas, T. E. Krikochoritis, and C. S. Tzafestas, "Robust sliding-mode control of nine-link biped robot walking," Journal of Intelligent and Robotic Systems, vol. 20, pp. 375-402, 1997.

[24] M. Nikkhah, H. Ashrafiuon, and F. Fahimi, "Robust control of underactuated bipeds using sliding modes," Robotica, vol. 25, pp. 367-374, 2007.

[25] M. Zohdy and A. Zaher, "Robust control of biped robots," Proc. The 2000 American Control Conference, vol. 3, pp. 1473-1477, 2000.

[26] C. S. O. B. G. Buche, "Control strategy for the robust dynamic walk of a biped robot," International Journal of Robotics Research, vol. 25, pp. 843-860, 2006.

[27] S. Kolathaya and A. D. Ames, "Exponential convergence of a unified clf controller for robotic systems under parameter uncertainty," in American Control Conference, 2014.

[28] B. Morris, M. J. Powell, and A. D. Ames, "Sufficient conditions for the Lipschitz continuity of QP-based multiobjective control of humanoid robots," Proc. 52nd IEEE Conf. Decision and Control, pp. 2920-2926, 2013.

[29] C. Chevallereau, G. Abba, Y. Aoustin, F. Plestan, E. R. Westervelt, C. Canudas-de-Wit, and J. W. Grizzle, "RABBIT: A testbed for advanced control theory," IEEE Control Systems Magazine, vol. 23, no. 5, pp. 57-79, October 2003.

[30] A. Isidori, Nonlinear Control Systems: An Introduction, 2nd ed. Berlin, Germany: Springer-Verlag, 1989.

[31] B. Morris and J. Grizzle, "Hybrid invariance in bipedal robots with series compliant actuators," in IEEE Conference on Decision and Control, San Diego, CA, USA, December 2006, pp. 4793 - 4800.

[32] — - "A restricted Poincaré map for determining exponentially stable periodic orbits in systems with impulse effects: Application to bipedal robots," in 44th IEEE Conference on Decision and Control, Seville, Spain, 2005.

[33] R. A. Freeman and P. V. Kokotović, Robust Nonlinear Control Design. Birkhäuser, 1996.

[34] Q. Nguyen and K. Sreenath. (2015) Optimal robust control for bipedal robots through control lyapunov function based quadratic programs. [Online]. Available: http://www.cmu.edu/me/hdr/publications/RSS15_full.pdf

[35] E. R. Westervelt, G. Buche, and J. W. Grizzle, "Experimental validation of a framework for the design of controllers that induce stable walking in planar bipeds," International Journal of Robotics Research, vol. 24, no. 6, pp. 559-582, June 2004.

[36] B. Morris, E. Westervelt, C. Chevallereau, G. Buche, and J. Grizzle, Fast Motions Symposium on Biomechanics and Robotics, ser. Lecture Notes in Control and Information Sciences. Heidelberg, Germany: Springer-Verlag, 2006, ch. Achieving Bipedal Running with RABBIT: Six Steps toward Infinity, pp. 277-297.

[37] H. Khalil, Nonlinear Systems - 3rd Edition. Upper Saddle River, NJ: Prentice Hall, 2002. 